\title{
Pharmacies des villes, pharmacies "par terre"
}

\section{Yannick Jaffré}

\section{(2) OpenEdition}

Journals

Édition électronique

URL : http://journals.openedition.org/apad/482

DOI : 10.4000/apad.482

ISSN : 1950-6929

Éditeur

LIT Verlag

Édition imprimée

Date de publication : 1 juin 1999

\section{Référence électronique}

Yannick Jaffré, «Pharmacies des villes, pharmacies "par terre" », Bulletin de l'APAD [En ligne], 17| 1999,

mis en ligne le 04 octobre 2006, consulté le 08 septembre 2020. URL : http://journals.openedition.org/ apad/482 ; DOI : https://doi.org/10.4000/apad.482

Ce document a été généré automatiquement le 8 septembre 2020.

Bulletin de l'APAD 


\title{
Pharmacies des villes, pharmacies "par terre"
}

\author{
Yannick Jaffré
}

1 Parler de soins en milieu urbain ne peut se faire sans aborder la question du médicament, puisqu'il est au cœur du dispositif de santé, allant de l'intimité de la consultation, où il soulage le mal et atteste de la vérité du diagnostic, jusqu'au "marché" où il correspond à de larges enjeux socio-économiques.

$2 \mathrm{Au}$ Mali, le domaine thérapeutique n'échappe pas à cette règle. La capitale, Bamako, dont la population est estimée aux alentours d'un million d'habitants sur une superficie de $240 \mathrm{~km}^{2}$, ne compte pas moins d'une centaine d'officines privées, et les dépenses publiques en médicaments pour les trois hôpitaux assurant la couverture sanitaire de cette communauté urbaine (G. Touré, Point G, Kati ), se sont élevées, en 1995, à 91 millions de francs CFA, soit 14,4\% du budget alloué à la santé (DNSP 1995). Enfin, pour l'ensemble du territoire national, le montant annuel des ventes est passé de 3,6 à 4,4 milliards de 1994 à 1997 (Anonyme 1998).

3 En ce pays, historiquement, les politiques du médicament ont évolué. Au lendemain de l'indépendance, de 1960 jusqu'en 1983, les médicaments et les soins sont gratuits. Puis, entre 1984 et 1989, dans le cadre de la stratégie des "soins de santé primaire", on a mis divers produits de base (antalgiques, antipyrétiques, antipaludéens, etc.) à la disposition des populations en confiant leur vente à des hygiénistes villageois. Les années comprises entre 1989 et 1994 virent ensuite se créer de nombreux dépôts pharmaceutiques gérés par des organisations non gouvernementales (ONG) et par diverses "associations communautaires". Une nouvelle étape s'est ouverte en 1994, puisque dans le cadre de l'Initiative de Bamako ${ }^{1}$, l'axe central de la politique pharmaceutique, outre un blocage temporaire des prix des médicaments et leur fixation consensuelle entre l'Etat, l'Ordre des pharmaciens, les syndicats et les grossistes ${ }^{2}$, est constitué par la promotion des produits génériques sous la forme de DCI (Dénomination Commune Internationale), dont le coût est moindre (Ballo et al. 1999). Enfin, soulignons que, depuis 1989, l'Etat soutient la création d'un nouveau type de structures sanitaires, les centres de santé communautaire (CSCOM), qui se 
caractérisent, tout au moins officiellement, par trois principes : ils sont créés par la population, ils fonctionnent pour la population, et sont gérés par la population. L'ensemble des vingt-huit CSCOM qui assurent la couverture sanitaire de la communauté urbaine de Bamako est approvisionné en médicaments essentiels par un groupement d'intérêt économique (GIE) dont le chiffre d'affaire, en 1997, était de l'ordre de 640 millions de FCFA, représentant une vente journalière allant de 1,5 à 2 millions de FCFA.

4 Ces politiques sanitaires ne peuvent cependant subsumer l'ensemble des pratiques sociales concernant le traitement de la maladie. Le quotidien toujours déborde ces textes et ces vouloirs officiels, et à Bamako, comme dans la plupart des villes africaines, chaque marché offre au chaland ses éventaires de feuilles, potions diverses et produits chimiques censés guérir de multiples maux. D'achats banals en modestes demandes, se constitue ainsi, en dehors des officines dûment répertoriées, un important secteur économique qui, pour ne prendre que l'exemple des achats des personnels de l'enseignement et de la culture, représentait en 1988 une dépense de l'ordre de 2 milliards de F CFA (Brunet-Jailly 1989). Mais si l'importance économique et sanitaire de ce secteur toléré par l'Etat ${ }^{3}$ est évidente, en comprendre le fonctionnement n'est pas chose facile.

5 Nous nous limiterons ici à décrire les ventes informelles de "médicaments chimiques" manufacturés en milieu urbain ${ }^{4}$. Pour ce faire, une piste s'offre à réduire nos égarements. Puisqu'il s'agit de commercer, ce nouement des offres et des demandes est, bien sûr, affaire de langage. Restent alors à décrire les interactions entre vendeurs et acheteurs, au plus proche des mots employés, restent à décrire les "termes" de l'échange, dans ces espaces urbains particuliers que sont les marchés ${ }^{5}$.

Les marchés, la vente et les distributeurs

6 Quelques marchés constituent le ventre de Bamako ${ }^{6}$. Depuis 1931, au centre de la ville, le "grand marché", ou "marché rose", avec une superficie de plus de 3000 m2,joue un rôle de distribution des marchandises vers des lieux de vente secondaires (Stele 1992). Cette vocation est aussi respectée pour ce qui concerne les produits pharmaceutiques puisqu'on y rencontre divers vendeurs "en gros" assurant le négoce avec des pays limitrophes comme le Nigéria, le Sénégal et la Guinée. A proximité se trouve le marché de dabanaani (lit. les quatre portes). Il offre sur ses étals de nombreuses denrées, où ferraille et outils voisinent avec légumes et viande. Au nord, le marché dibida (lit. la porte des rôtisseries) est le principal centre de commercialisation des légumes et du poisson. Enfin, d'implantation plus récente, celui de rayida (lit. proche des rails) se confond avec les nombreuses échoppes qui cernent l'Assemblée Nationale.

7 Sur l'ensemble de ces marchés, 197 vendeurs proposent plus de 50 sortes de produits pharmaceutiques, dont les prix "au comprimé" s'échelonnent de 50 de FCFA à 200 de FCFA. L'observation méthodique ${ }^{7}$ de la vente réalisée par ces "pharmaciens informels" a permis d'estimer en moyenne le chiffre d'affaire à 3500 de FCFA par heure et par vendeur. Si l'on s'accorde sur ces estimations, ces sommes représentent pour l'ensemble des commerçants des marchés étudiés des échanges quotidiens se situant aux alentours de 10 millions de FCFA et donc, annuellement, un flux monétaire de plus de 2 milliards, pour ces quelques marchés de la seule capitale!

8 Mais si l'affaire est d'importance, les vendeurs restent d'apparence modeste. Ils sont généralement analphabètes et sans formation médicale particulière. Leurs connaissances consistent avant tout en divers savoir-faire: "tours de mains" 
indispensables, tant pour acheter les produits que pour les prescrire. Trois principaux types d'apprentissage, correspondant à trois modes de légitimation de leur pratique professionnelle, dominent leurs propos. Un premier ensemble d'interlocuteurs a appris ce travail auprès d'un membre de la famille jouissant d'un statut particulier, et se réclame ainsi de parents "chasseurs-magiciens" (dansa), "féticheurs" (balitigi) ou géomanciens (cinyèndala), qui, tout autant que des connaissances, leur ont transmis une préoccupation ou un droit de s'occuper de la santé physique et morale de ceux qui en formulent la demande. Un autre groupe témoigne des vertus formatives de l'amitié : teriya ka di ni balimaya ye (l'amitié vaut mieux que la famille), ce qui signifie que leur apprentissage s'effectua au sein d'un des réseaux des nouvelles convivialités urbaines : "promotionnaires", compagnons "d'exode" ou de "grin" (groupe de thé et de discussion), etc. Enfin l'élection, manifestée par un rêve ou la sensation d'être désigné, constitue la dernière forme d'acquisition d'une légitimité thérapeutique.

9 A l'évidence, il ne s'agit aucunement de spécialisations ou de "filières locales de formation", et, s'il y a naissance d'une nouvelle profession urbaine du "secteur informel", elle concerne plutôt l'acquisition d'aptitudes commerciales - connaître notamment les réseaux pour se procurer des produits - que la maîtrise et la volonté de progresser dans les domaines du diagnostic et de la thérapeutique ${ }^{8}$. Par ailleurs, à l'inverse de compétences spécifiques scientifiquement fondées, les attestations de légitimité s'accumulent plus qu'elles ne s'excluent, même si, par exemple, ceux qui vendent des produits végétaux soulignent avant tout les aspects "traditionnels" de leur fonction, alors que ceux qui proposent des médicaments manufacturés évoquent préférentiellement la modernité des savoirs acquis lors de séjours à l'étranger. L'unanimité est acquise cependant pour ce qui concerne la médecine moderne officielle. Pour tous, leurs pratiques et la médecine moderne ne seraient pas antagonistes, mais complémentaires: "Nous n'avons pas de machine pour voir à l'intérieur de l'homme. Notre travail se limite à l'imagination (kungolo baara, lit. travail de la tête). Si nous posons des questions à un malade, s'il n'arrive pas à nous donner une réponse satisfaisante, on l'envoie chez les médecins. Il y a une complémentarité entre nous et les pharmaciens (anw ni faramansi mogow bé nyogon dafa)" (un jeune vendeur). Propos lénifiants sans aucun doute, minimisant les conflits et les risques liés à la vente de produits officiellement prohibés. Reste que cette conception correspond très largement aux conduites les plus communes des populations, qui fort pragmatiquement "tentent leur chance sanitaire" dans le secteur formel tout autant qu'informel $^{9}$.

Les remèdes

10 Pour la santé publique, outre connaître la composition chimique de ces produits illicites et leurs éventuels risques, il est important de comprendre les ressorts de leurs succès. Cette "entrée par les médicaments" oblige à quitter un instant des yeux l'interaction médecin-malade souvent considérée comme centrale dans la relation thérapeutique. Plus exactement, de la repositionner dans un écheveau large de relations qui associent les laboratoires de la recherche à la fabrication et à la commercialisation des médicaments, les pharmaciens, les médecins et les patients, mais également d'autres médiateurs, comme les journaux, les livres, les membres d'une famille, les collègues, les amis, les relations, etc. (Akrish 1995). Bref, les rumeurs autour de l'efficacité d'un remède, le nom donné au produit, sa présentation, les vertus thérapeutiques qu'on lui accorde - et qu'importe, dès lors que la croyance est partagée, 
que ce soit à tort ou à raison - sont à prendre en compte pour comprendre le jeu complexe des offres et des demandes.

11 Aux étals des "pharmacies par terre", une cinquantaine de produits "thérapeutiques" manufacturés sont exposés aux acheteurs. Sans viser à une impossible exhaustivité, puisque de nouveaux produits apparaissent sans cesse, nous présentons un tableau mettant en rapport le produit, sa dénomination populaire et la pathologie ressentie qu'il est supposé soigner.

\begin{tabular}{|c|c|c|c|}
\hline $\begin{array}{l}\text { Nom indiqué sur } \\
\text { le produit }\end{array}$ & Dénomination populaire & $\begin{array}{l}\text { Prix } \\
\text { moyen de } \\
\text { vente à } \\
\text { l'unité }\end{array}$ & $\begin{array}{l}\text { Pathologies ressenties } \\
\text { « soignées » par ces produits }\end{array}$ \\
\hline Ampicilline 500 & $\begin{array}{l}\text { ampisilini fununfura } \\
\text { (médicament de l'« enflure ») }\end{array}$ & $75 \mathrm{Cfa}$ & $\begin{array}{l}\text { funun (enflure) } \\
\text { joli (plaie) }\end{array}$ \\
\hline Amoxicilline & amosisilin & $75 \mathrm{Cfa}$ & $\begin{array}{l}\text { funun kònòbòli (diarrhée, lit. } \\
\text { ventre qui court) }\end{array}$ \\
\hline Aspirine UPSA & jiwuli (eau qui bout) & $50 \mathrm{Cfa}$ & $\begin{array}{l}\text { mura farigan (fièvre du } \\
\text { rhume) } \\
\text { sumaya (fraicheur, traduit } \\
\text { fréquemment par paludisme) }\end{array}$ \\
\hline Aspirine 500 & $\begin{array}{l}\text { aspirini farigan fura (médicament } \\
\text { du corps chaud) }\end{array}$ & $50 \mathrm{Cfa}$ & $\begin{array}{l}\text { kundimi (maux de tête) } \\
\text { farigan (corps chaud) }\end{array}$ \\
\hline Auréomycine $1 \%$ & $\begin{array}{l}\text { nyèna tulunin (petite huile de } \\
\text { l'œil) }\end{array}$ & $200 \mathrm{cfa}$ & nyèdimi (mal aux yeux) \\
\hline Auréomycine $2 \%$ & $\begin{array}{l}\text { joli tulunin (petite huile pour la } \\
\text { plaie) }\end{array}$ & $200 \mathrm{Cfa}$ & $\begin{array}{l}\text { joli (plaies) } \\
\text { "muso jalen» (lit. femme } \\
\text { sèche, sans sécrétions } \\
\text { vaginales) }\end{array}$ \\
\hline $\begin{array}{l}\text { B complexe } \\
\text { vitamine }\end{array}$ & $\begin{array}{l}\text { dabòsifuranin (médicament pour } \\
\text { les plaies de la bouche) }\end{array}$ & $5 \mathrm{Cfa}$ & dabòsi (plaies dans la bouche) \\
\hline CAC 1000 & jiwuli (eau qui bout) & $175 \mathrm{Cfa}$ & sègèn (fatigue) \\
\hline Chloroquine 100 & niwakinin & $10 \mathrm{Cfa}$ & sumaya \\
\hline BF 500 & $\begin{array}{l}\text { berebila jèman (laisser le bâton, } \\
\text { blanc) }\end{array}$ & $50 \mathrm{Cfa}$ & $\begin{array}{l}\text { dimiw bè̀ (toutes les } \\
\text { douleurs) }\end{array}$ \\
\hline $\begin{array}{l}\text { Chloroquine } \\
\text { (gélule) }\end{array}$ & $\begin{array}{l}\text { kunbilènin jèman (tête rouge, } \\
\text { blanche) }\end{array}$ & $15 \mathrm{Cfa}$ & kònòbòli \\
\hline Daga & dakan (lit. destin) & $25 \mathrm{Cfa}$ & sumaya \\
\hline
\end{tabular}




\begin{tabular}{|c|c|c|c|}
\hline Dependol & kundimifura & $10 \mathrm{Cfa}$ & kundimi \\
\hline Dexamethasone & dafurukubanin (les grosses joues) & $10 \mathrm{Cfa}$ & bònya (grossir) \\
\hline Ephédrine & $\begin{array}{l}\text { misèmanin (le petit), katorzi } \\
\text { (quatorze), dèn kèlèn dèmè baga } \\
\text { (la seule chose qui peut t'aider) }\end{array}$ & & sègèn \\
\hline Falbitone & bobarabanin (grosses fesses) & $50 \mathrm{Cfa}$ & $\begin{array}{l}\text { jolicaya (augmenter le sang) } \\
\text { bonya }\end{array}$ \\
\hline Indométacine & fasafura & $10 \mathrm{Cfa}$ & $\begin{array}{l}\text { fasadimi } \quad \text { (douleurs } \\
\text { musculaires, ligamentaires, } \\
\text { etc.) }\end{array}$ \\
\hline Ladinax & $\begin{array}{l}\text { kònòdimifura muso ka kònòdimi } \\
\text { furanin (médicament pour les } \\
\text { maux de ventre des femmes) }\end{array}$ & $50 \mathrm{Cfa}$ & kònòdimi \\
\hline Périactine & $\begin{array}{l}\text { bonyafuranin (médicament pour } \\
\text { grossir) } \\
\text { sunògòfura (médicament pour } \\
\text { dormir) }\end{array}$ & $50 \mathrm{Cfa}$ & fasa sunògòbaliya (insomnie) \\
\hline Stopache & $\begin{array}{l}\text { bèrèbila nèrèmuguman (laisser le } \\
\text { bâton, jaune) }\end{array}$ & $50 \mathrm{Cfa}$ & $\begin{array}{l}\text { kòdimi (maux de dos) } \\
\text { fasadimi } \quad \text { (douleurs } \\
\text { musculaires) }\end{array}$ \\
\hline Stomach & kònòdimifura & $50 \mathrm{Cfa}$ & kònòdimi \\
\hline Tétracyline & kunbilènin (tête rouge) & $15 \mathrm{Cfa}$ & funun kònòbòli nyèdimi joli \\
\hline $\begin{array}{l}\text { Yan duniya } \\
\text { (expression } \\
\text { hawsa) }\end{array}$ & $\begin{array}{l}\text { sumusumu (causerie intime, } \\
\text { embrasser) }\end{array}$ & & aphrodisiaque \\
\hline
\end{tabular}

12 A travers cette présentation tabulaire, nos étalages en viennent à constituer un corpus "pharmaco-linguistique" où se mêlent douleurs, mots et comprimés (fura kisè, lit. grain de médicament), dévoilant une sorte de très efficace "merchandising" local révélateur de quelques modes populaires d'appropriation du remède et d'intégration cognitive des savoirs biomédicaux. Et puisque commercer est aussi une manière d'accommoder les signes, essayons maintenant de comprendre quelques règles de cette "sémantisation de l'objet" thérapeutique (Barthes 1985).

13 Au plus simple, un premier mode de nomination du remède correspond à un emprunt (ampisilinin, niwakini) ou à une sorte de rapprochement interprétatif. Ainsi, par exemple, un terme inconnu comme "daga" est-il prononcé "dakan" (le destin), cette modification des phonèmes permettant aux acheteurs de donner sens à ce produit : "il est le destin de la maladie, puisqu'il la tue". 
14 Viennent ensuite de simples descriptions. La gélule de couleur rouge est "tête rouge" (kunbilènin), le produit effervescent "bout" au contact de l'eau (jiwuli), et les pommades sont huileuses (tulunin).

Un troisième procédé concerne les maux banals et quotidiens, désignés le plus souvent par un terme correspondant à une description sémiologique ${ }^{10}$. Les traitements concernant ces affections sont fréquemment nommés par simple adjonction du suffixe fura (médicament) au nom de la maladie. Quelques exemples sont ici éloquents : à kònòdimi (maux de ventre) correspond un traitement nommé kònòdimifura, à kundimi (maux de tête) kundimifura, etc. Procédés simples au regard du linguiste, ces créations lexicales intimement mêlées à des représentations de la maladie ne sont pas sans conséquences pour la santé publique. Associées à d'autres déterminants, économiques notamment, elles induisent des démarches de soin où les traitements ne sont bien souvent que symptomatiques.

Un autre procédé de nomination du remède s'applique à en décrire les effets. Ainsi la dexaméthasone ou le falbitone qui sont des corticoïdes ont comme effet secondaire de provoquer une fausse obésité, donnant l'apparence de formes girondes. Ces produits sont nommés dafurukubanin (grosses joues) ou bobarabanin (grosses fesses) et s'appliquent à procurer des attraits plus charnus à celles qui s'en croient dépourvues. De même, les effets excitants de l'éphédrine, et notamment l'insomnie qu'elle provoque, en font une "drogue" accessible, se présentant parfois comme la "seule chose qui peut t'aider". Enfin, les antalgiques aux immédiats et très larges effets permettent au malade de "laisser le bâton" qui le soutenait.

17 Ces modestes jeux linguistiques révèlent les préoccupations sanitaires, esthétiques, ou psychologiques des populations. Mieux que lors d'enquêtes d'opinion, la demande sanitaire s'y révèle en acte, construisant parfois de complexes polysémies thérapeutiques comme lorsque l'auréomycine, outre ses qualités antibiotiques, devient un produit lubrifiant, permettant à certaines femmes de simuler l'envie de rapports sexuels. Mais ces tropes "pharmaco-sémantiques" éclairent aussi un des modes d'appropriation de l'information sanitaire. Privés de conseils médicaux et du savoir nécessaire permettant de comprendre la composition du produit et les spécificités de son usage, les consommateurs ne peuvent se fier qu'aux effets qu'ils observent. Autrement dit, dans ce mode de raisonnement et d'évaluation de l'efficacité du produit, le référent n'est pas la molécule chimique active, mais l'effet ressenti ${ }^{11}$ ou attendu, puisque souvent ils se confondent dans ce que l'on appelle "l'effet placebo".

18 En ce domaine où l'on consomme le symbole autant que le produit, les figurations dessinées sur les emballages sont essentielles. A l'inverse des produits génériques présentés sous une forme neutre - comprimés blancs dans un étui de plastique transparent -, elles permettent soit de visualiser le mal en le localisant corporellement. soit d'anticiper les effets du traitement. Bref, ces médicaments "par terre" sont avant tout des "médicaments de proximité culturelle", instaurant une continuité presque parfaite entre une pathologie ressentie, son traitement, et une figuration iconographique immédiatement comprise.

Plaintes, traitements, vendeurs et acheteurs

Cette proximité se révèle aussi dans les interactions entre vendeur et client, lors de l'achat du traitement. Nombre d'acheteurs évoquent des raisons pécuniaires, liées surtout à des possibilités d'achat à l'unité, pour expliquer leur choix : "Si tu vois que je viens d'acheter les médicaments au marché, c'est parce que les produits y sont moins 
chers que ceux de la pharmacie. Par exemple, si j'ai cent francs, je peux acheter au marché, deux comprimés de nivaquine et je garde le reste de mon argent" (jeune acheteur au Grand Marché).

D'autres raisons cependant se conjuguent. La première résulte de la correspondance directe entre ces produits et les pathologies ressenties auto-diagnostiquées : "J'achète kunfinin contre furudimi. Quand je prends kunfinin, ça calme ma douleur et je dors bien. Je prends aussi kunbilènin contre le kònòna jòli ..." (vieille femme, marché Dabanaani). Bref, au marché, c'est à chaque maladie/symptôme son traitement, alors que les pharmacies obligent à des démarches complexes, et notamment obtenir une prescription médicale rédigée en français et devant être présentée en un lieu culturellement mal maîtrisé, où les possibilités de tri et d'achat des produits à l'unité sont réduites. De plus, au cabinet médical comme à l'officine, le diagnostic et l'ordonnance se réfèrent à des catégories bio-médicales qui fondées sur l'étiologie de la maladie, ne peuvent correspondre ni aux découpages sémiologiques qui gouvernent en grande partie les remèdes populaires, ni s'appliquer à des pathologies supposées avoir une origine magico-religieuses, voire a fortiori, à des maladies que l'on traite sans les connaître (bana sidonbali, lit. maladies non-identifiées).

Par ailleurs, et c'est là une autre raison de succès, l'espace social du marché semble protéger, mieux que les services de santé, la confidentialité et la pudeur des acheteurs. Paradoxalement, le brouhaha du marché préserve le secret, et certaines personnes, qui ont honte d'aller à l'hôpital pour expliquer leur maladie, préfèrent l'anonymat et la rapidité de la vente locale: "La maladie dont je souffre me donne honte d'aller à l'hôpital, car si tu pars à l'hôpital, tu seras déshabillée, avant d'être consultée. S'il y a plusieurs personnes dans la salle. elles participent toutes à la consultation..." (jeune fille, Grand Marché). "J'ai acheté sumusumu (lit. embrasser) avec des vendeurs haoussa. Ce sont des produits qui stimulent le désir chez l'homme. Il faut en mettre un peu dans le vagin avant les rapports sexuels. Je mets aussi de l'auréomycine, ça facilite la pénétration et donne une grande satisfaction pendant les rapports... J'ai connu tous ces produits par l'intermédiaire d'une amie, sinon j'ai honte d'aller à l'hôpital pour cela" (jeune femme, Dibida).

Les communautés de langue et de représentations de la maladie, la connaissance des codes réglant les interactions marchandes, et le partage des mêmes sociabilités entre vendeur et client, font du marché un prolongement public de l'espace privé, créant ainsi une continuité entre le plus intime d'une demande et le plus inconnu d'un traitement. Concrètement, rien et pas même le comptoir du pharmacien, ne sépare celui qui vend de celui qui demande. Le marché est un lieu de proximité, aux remèdes sémiologiquement adaptés.

En Afrique et au Mali comme ailleurs, le marché délimite un espace d'échanges spécifiques, où l'on commerce au sens ancien du terme, en mêlant les mots et l'argent. D'un point de vue sanitaire, ces convenances partagées, et les interactions qui s'y déploient, révèlent "en acte" des rapports populaires au corps et à la maladie. Ce "savoir faire de la rencontre", cette quotidienneté banale, charrie cependant, d'un point de vue de santé publique, nombre d'importantes questions comme celles de l'accès aux soins, de l'observance ${ }^{12}$ des traitements, ou de l'utilisation de certaines drogues. multiples échange sociaux, le marché dissimule autant qu'il montre. Dans le domaine 
thérapeutique, il cache derrière quelques produits les plaintes des populations les plus démunies et les difficultés de l'Etat à y répondre.

\section{BIBLIOGRAPHIE}

Ankri J., Le Disert D., Henrad J.C. 1993. "Comportements individuels face aux médicaments, de l'observance thérapeutique à l'expérience de la maladie, analyse de la littérature". Santé Publique $4: 427-441$.

Akrich, M. 1995. "Petite anthropologie du médicament". Techniques et Cultures 25-26. Anonyme 1998. Document préparatoire de la troisième rencontre des Ministres de la santé des pays de la zone franc et des pays associés sur la politique du médicament. Libreville Gabon.

Assi Gbonon, R. 1992. Médicaments vendus dans les marchés en Côte d'ivoire. Doc. ronéoté, Union Européenne.

Ballo, M B., Boundy F., Diakité B.D. 1999. initiative de Bamako, plus de 10 ans après. Bamako : MSSPA (Ministère de la Santé, de la Solidarité et des Personnes Agées).

Barthes, R. 1985. "Sémantique de l'objet". In L'aventure sémiologique, Paris : Editions du Seuil : 249-260.

Brunet-Jailly, J. 1988. Financement des coûts récurrents de la santé au Mali. Genève : OMS.

Diakité, B.D., Diarra T., Traoré S. 1993. "Recours aux soins et consommation médicale dans le quartier de Bankoni". In : Brunet-Jailly (ed). Se soigner au Mali, Paris, Karthala : 155-175

Fassin, D. 1985. "Du clandestin à l'officine, les réseaux de vente illicite des médicaments au Sénégal", Cahiers d'Etudes Africaines 98 : 161-177.

Jaffré, Y. \& Olivier de Sardan, J-P. 1999. La construction sociale des maladies. Les entités nosologiques populaires en Afrique de l'Ouest. Paris : PUF.

Stele, J. 1992. Rénovation des marchés centraux du district de Bamako, Etude de faisabilité. Rapportfinal. Bamako : "Groupe des huit"-SNED.

\section{NOTES}

1.Cette stratégie, adoptée en septembre 1987 à Bamako, lors de la $37^{\text {ème }}$ réunion du comité régional de l'OMS, par les ministres africains de la santé, est caractérisée par quatre axes : étendre la couverture géographique des services de santé de base, associer les bénéficiaires à la création et à la gestion des services de santé de base, développer l'accès au traitement par la promotion des médicaments DCI, instituer une politique de recouvrement des coûts.

2.Les efforts en ce domaine sont importants. Le gouvernement a ainsi réduit la pression fiscale sur les médicaments de $22 \%$ à $6 \%$, les grossistes ont diminué leur marge bénéficiaire de $20 \%$ à $4 \%$, et les pharmaciens d'officine de $25 \%$ à $20 \%$. Les médicaments essentiels génériques ont quant à eux été totalement détaxés. 
3.C'est par exemple ce que souligne Fassin (1985), à propos du Sénégal : "La tolérance des pouvoirs publics vis-à-vis des vendeurs est évidente. Sur les marchés. dans les rues. autour des places, ils circulent librement, s'installent sans obstacle. Le meilleur indice de leur présence est l'acquittement quotidien d'une patente (40 à 75 FCFA selon la taille de l'emplacement) auprès du juuti (de l'anglais duty) de la mairie qui leur reconnaît ainsi non seulement une existence mais aussi un espace ; à cette taxe s'ajoutent les 25 FCFA versés au gardien du marché. Ces deux prélèvements concernent les vendeurs de médicaments au même titre que n'importe quel commerçant du marché".

4.Nous remercions Alou Dembele et Adrienne Samake qui ont effectué les enquêtes. 5.Signalons que L. Chillio a étudié dans une perspective similaire la vente de médicaments à Niamey (thèse en cours)

6.Essayant de rendre compte du cours normal des choses, nous avons volontairement exclu de notre étude un autre marché de la capitale, celui de Médine, où une partie de la vente des "produits traditionnels" a été réorganisée par une association locale et une ONG étrangère.

7.Nous avons compté systématiquement et à plusieurs reprises les vendeurs présents à différentes heures sur ces marchés. Nous les avons ensuite indexés sous un numéro, puis avons tiré au hasard une vingtaine d'entres eux. Nous avons pratiqué de même pour les jours et les heures d'observation où nous avons systématiquement relevé le nombre des clients, les produits vendus et les sommes encaissées.

8.De semblables remarques ont été faites par Assi Gbonon (1992) pour la Côte d'Ivoire : "C'est un nouveau métier qui s'organise et cherche à se structurer en essayant de mettre en place un véritable réseau de distribution avec les grossistes, les détaillants, les livreurs et les colporteurs". Selon la même enquête il y a trois sources d'approvisionnement en médicaments. $48 \%$ de ces médicaments proviennent des grossistes installés à Abidjan. La deuxième source d'approvisionnement est constituée par les pharmaciens ( $31 \%$ ), chez qui les trafiquants font leurs achats. La troisième est constituée par les livreurs inconnus ou occasionnels (22\%).

9.Dans un quartier périphérique de cette même ville, Diakité, Diarra et Traoré remarquent que « sous toutes ses formes, l'automédication est souvent le premier recours " (1993).

10.Pour des entités nosologiques populaires plus complexes, cf. Jaffré \& Olivier de Sardan 1999.

11.Ce qui ne va pas sans comporter de réels risques sanitaires, comme avec l'utilisation des produits corticoïdes.

12.Sur cette question, nous renvoyons à l'article de synthèse d'Ankri et al. (1995).

\section{AUTEUR}

\section{YANNICK JAFFRÉ}

EHESS-CNRS. Centre de la Vieille Charité, 13002 Marseille. Tel : +33491 140772. http :// ehess.cnrs-mrs.fr/shadyc/accueil.html 